\title{
A Self-triggered Visual Servoing Model Predictive Control Scheme for Under-actuated Underwater Robotic Vehicles
}

\author{
Shahab Heshmati-Alamdari, Alina Eqtami, George C. Karras, Dimos V. Dimarogonas \\ and Kostas J. Kyriakopoulos
}

\begin{abstract}
This paper presents a novel Vision-based Nonlinear Model Predictive Control (NMPC) scheme for an underactuated underwater robotic vehicle. In this scheme, the control loop does not close periodically, but instead a self-triggering framework decides when to provide the next control update. Between two consecutive triggering instants, the control sequence computed by the NMPC is applied to the system in an openloop fashion, i.e, no state measurements are required during that period. This results to a significant smaller number of requested measurements from the vision system, as well as less frequent computations of the control law, reducing in that way the processing time and the energy consumption. The image constraints (i.e preserving the target inside the camera's field of view), the external disturbances induced by currents and waves, as well as the vehicle's kinematic constraints due to under-actuation, are being considered during the control design. The closed-loop system has analytically guaranteed stability and convergence properties, while the performance of the proposed control scheme is experimentally verified using a small underactuated underwater vehicle in a test tank.
\end{abstract}

\section{INTRODUCTION}

Visual Servo Control has been extensively used in the past for the autonomous operation of underwater robotic vehicles. Complex missions such as ship hull inspection, surveillance of underwater facilities (e.g oil platforms) and handling of underwater equipment (e.g control panels, valves) require detailed and continuous visual feedback which can be obtained from either monocular or stereo vision systems.

In a standard underwater vehicle configuration, the camera is directly mounted on the robot (eye-in-hand camera system) and the control error can be in general formulated in three different ways: (i) Position-Based Visual Servoing (PBVS), where the visual features extracted from the image are used to estimate the 3D pose of the robot wrt the target; (ii) ImageBased Visual Servoing (IBVS), where the control inputs are determined directly on the 2D image plane based on the error of the image features between the current and desired images, and (iii) Hybrid Visual Servoing, where 3D PBVS is combined with 2D IBVS [1],[2]. The motion control of an

Shahab Heshmati-Alamdari, George C. Karras and Kostas J. Kyriakopoulos are with the Control Systems Lab, Department of Mechanical Engineering, National Technical University of Athens, 9 Heroon Polytechniou Street, Zografou 15780, Greece \{shahab, karrasg, kkyria@mail.ntua.gr\}. Dimos V. Dimarogonas is with the KTH ACCESS Linnaeus Center and the KTH Centre for Autonomous Systems, School of Electrical Engineering, Royal Institute of Technology (KTH), Stockholm, Sweden $\{$ dimoslee.kth.se\}. His work was supported by the Swedish Research Council and the EU HYCON2 NoE. Alina Eqtami is with Cardiovascular Surgery, Childrens Hospital Boston, Harvard Medical School, Boston MA, 02115, USA. \{alina.eqtami@childrens.harvard.edu\}.

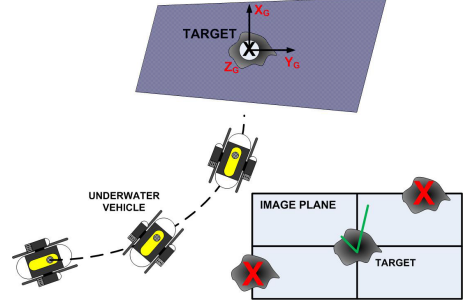

Fig. 1: Problem Statement: Navigation and stabilization of the vehicle wrt a visual target while maintain target inside camera's optical field.

underwater vehicle is generally a highly nonlinear problem. Moreover, real applications (e.g inspection and surveillance tasks) impose constraints on the inputs and the states of the vehicle (i.e visual and/or kinematic constraints). Nonlinear Model Predictive controllers can efficiently handle nonlinearities and constraints simultaneously [3] . However, as NMPC schemes constitute in solving a constrained Optimal Control Problem (OCP) at each instant, they are considered to be computationally demanding. This motivates the event-based design framework of the predictive controllers in order to compute the control law only when is needed. The eventbased formulation of control tasks is a quite recent development that can lead to the alleviation of energy consumption as well as to the improvement of the requirements of the computational resources. Under this formulation the decision for the control update is based on a certain condition of the state of the system which can lead not only to a more flexible aperiodic sampling but can also guarantee to preserve necessary properties of the system such as stability and convergence, [4]. More specifically, in the case of selftriggered control only the latest state measurement needs to be known for determining the next triggering instant. Some relevant results for the self-triggered set-up can be found in [5],[6], [7].

In this paper, a Self-triggered Position Based Visual Servo NMPC scheme for the motion control of an under-actuated underwater vehicle is presented. The purpose of this work is to navigate and stabilize the vehicle towards a visual target by using the proposed control framework and assuring that the target will always remain inside the camera's field of view (Fig. 1). The filed of view (FOV) constraints are handled by the explicit formulation of the visual constraints inside the NMPC framework. The main contribution of this work relies in the design of a framework that will provide aperiodic control sequences that lead to stable closed-loop responses and of a mechanism that will decide when these 


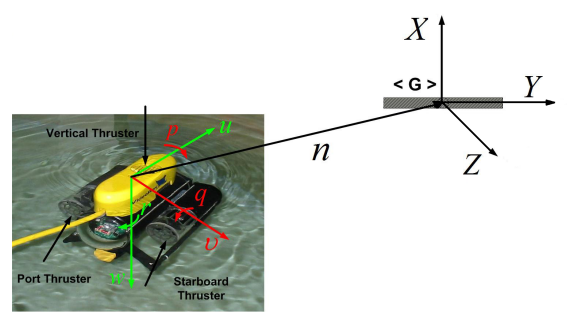

Fig. 2: Differential Drive Robot. Red color indicates no actuation availability, while green color indicates actuation availability along body frame axes.

control updates should occur. This results to the reduction of the computational effort and the energy consumption which are of utmost importance especially in the case of Autonomous Underwater Vehicles (AUVs) where lean computational algorithms and low energy consumption are required. Some preliminary results on the self-triggered MPC for nonholonomic systems are given in [8].

Experimental results of this kind of event-based formulations are scarce in the literature,[9], [10], and to the best of our knowledge this event-based set-up has never been used before to control a real underwater vehicle. The performance of our proposed control scheme is experimentally verified using a small under-actuated underwater vehicle. The experimental results are quite satisfying as the vehicle's state vector converges to a bounded set around the desired position and the triggering instants are significantly reduced with respect to (wrt) the traditional time-triggered case. The remainder of the paper is organized as follows: In Section II, the modeling of the underwater vehicle with the imposed constraints and uncertainties, the vision-based state estimation of the vehicle, as well as the whole problem statement are presented. Section III accommodates the robust stability analysis for the NMPC scheme which leads to the self-triggered framework. Section IV, illustrates the efficiency of the proposed approach through a set of experimental results. Finally, Section V concludes the paper.

\section{Problem Formulation}

\section{A. Mathematical Modeling}

Generally an underwater vehicle is considered as a 6 DOF free body with position and Euler angle vector $\mathbf{x}=$ $\left[\begin{array}{llllll}x & y & z & \phi & \theta & \psi\end{array}\right]^{T}$. The body velocities vector is defined as $\mathbf{v}=\left[\begin{array}{lllll}u & v & w & p & q\end{array}\right]^{T}$ where the components are surge, sway, heave, roll, pitch and yaw respectively (Fig.2). The general form of the kinematic equations of an underwater vehicle is given [11]:

$$
\dot{\mathbf{x}}=\mathbf{J}(\mathbf{x}) \mathbf{v}
$$

where: $\mathbf{J}(\mathbf{x})$ is the Jacobian matrix transforming the velocities from the body-fixed to earth-fixed frame. The vehicle used in this work is a 3 DOF VideoRay Pro ROV. It is equipped with three thrusters, which are effective only in surge, heave and yaw motion (Fig.2), meaning that the vehicle is under-actuated along the sway axis. The angles $\phi, \theta$ and angular velocities $p$ and $q$ are negligible and we can consider them to be equal to zero. Due to the above assumption and the relative low speed of the vehicle, we only consider the kinematic model of the vehicle, which in its discrete-time form is formulated as follows [11]:

$$
\begin{gathered}
x_{k+1}=f\left(x_{k}, V_{k}\right)+g\left(x_{k}, v_{k}\right) \Rightarrow\left[\begin{array}{l}
\chi_{k+1} \\
y_{k+1} \\
z_{k+1} \\
\psi_{k+1}
\end{array}\right]= \\
\left.=\left[\begin{array}{c}
\chi_{k} \\
y_{k} \\
z_{k} \\
\psi_{k}
\end{array}\right]+\left[\begin{array}{ccc}
\cos \psi_{k} & 0 & 0 \\
\sin \psi_{k} & 0 & 0 \\
0 & 1 & 0 \\
0 & 0 & 1
\end{array}\right]\left[\begin{array}{c}
u_{k} \\
w_{k} \\
r_{k}
\end{array}\right]+\left[\begin{array}{c}
-\sin \psi_{k} \\
\cos \psi_{k} \\
0 \\
0
\end{array}\right] v_{k}\right] d t
\end{gathered}
$$

where $x=\left[\chi_{k}, y_{k}, z_{k}, \psi_{k}\right]^{\top}$ denotes the state vector at the time-step $k$ which includes the position and orientation of the vehicle wrt the target frame $\mathcal{G}$. The control input of the system is $V_{k}=\left[u_{k}, w_{k}, r_{k}\right]^{\top}$ and $d t$ denotes the sampling period. In [12], it was proven that for any vehicle described by (2), for any bounded control input $\left[u_{k}, r_{k}\right]$ the velocity about the sway direction $v_{k}$ can be seen as a bounded perturbation with upper bound $\left\|v_{k}\right\| \leq \bar{v}$ that vanishes at the point $x=0$. Consequently, this point is an equilibrium of the kinematic system of (2). Note that throughout this paper the notation $(\cdot)$ will denote the upper bound for each of the variables. Therefore we consider the system:

$$
\begin{aligned}
& x_{k+1}=f\left(x_{k}, V_{k}\right) \Rightarrow \\
& {\left[\begin{array}{l}
\chi_{k+1} \\
y_{k+1} \\
z_{k+1} \\
\psi_{k+1}
\end{array}\right]=\left[\begin{array}{l}
\chi_{k} \\
y_{k} \\
z_{k} \\
\psi_{k}
\end{array}\right]+\left[\begin{array}{ccc}
\cos \psi_{k} & 0 & 0 \\
\sin \psi_{k} & 0 & 0 \\
0 & 1 & 0 \\
0 & 0 & 1
\end{array}\right]\left[\begin{array}{l}
u_{k} \\
w_{k} \\
r_{k}
\end{array}\right] d t}
\end{aligned}
$$

As the nominal kinematic system of the underwater vehicle. The function $g(\cdot)$ is considered as a bounded inner disturbance of the system that vanishes at the origin. Also, $g\left(x_{k}, v_{k}\right) \in \Gamma \subset \mathbb{R}^{4}$ with $\Gamma$ to be a compact set, such that:

$$
\left\|g\left(x_{k}, v_{k}\right)\right\| \leq \bar{\gamma} \quad \text { with } \quad \bar{\gamma} \triangleq \bar{v} d t
$$

The robot moves under the influence of an irrotational current which behaves as an external disturbance. The current has components wrt the $\chi, y$ and $z$ axes, denoted by $\delta_{\chi}, \delta_{y}$ and $\delta_{z}$ respectively. Also it has a slowly-varying velocity $\delta_{c}$ which is upper bounded by $\left\|\delta_{c_{k}}\right\| \leq \bar{\delta}_{c}$ and it has direction $\beta$ in $\chi-y$ plane and $\alpha$ in $z$ axes, wrt the global frame, see Fig.3. In particular we set $\delta_{k}=\left[\delta_{(\chi / k)}, \delta_{(y / k)}, \delta_{(z / k)}, 0\right]^{\top} \in \Delta \subset \mathbb{R}^{4}$ with $\Delta$ to be a compact set, where:

$$
\begin{aligned}
& \delta_{(\chi / k)} \triangleq \delta_{c_{k}} \cdot \cos \beta \cdot \sin \alpha \cdot d t \\
& \delta_{(y / k)} \triangleq \delta_{c_{k}} \cdot \sin \beta \cdot \sin \alpha \cdot d t \\
& \delta_{(z / k)} \triangleq \delta_{c_{k}} \cdot \cos \alpha \cdot d t
\end{aligned}
$$

It is easy to show that $\left\|\delta_{k}\right\| \leq \bar{\delta}$, with $\bar{\delta}=\bar{\delta}_{c} \cdot d t$. Taking into consideration the aforementioned disturbances that affect the vehicle, we are now ready to model the perturbed system as follows:

$$
x_{k+1}=f\left(x_{k}, V_{k}\right)+\omega_{k}
$$

with $\omega_{k}=g\left(x_{k}, v_{k}\right)+\delta_{k} \in \Omega \subset \mathbb{R}^{4}$ as the result of adding the inner and external disturbances of the system. $\Omega$ is a compact set such that $\Omega=\Delta \oplus \Gamma$, where " $\oplus$ " denotes the Minkowski addition of two sets. Since the sets $\Delta$ and $\Gamma$ are compact, it yields that $\Omega$ is also a compact set, bounded by 
$\left\|\omega_{k}\right\| \leq \bar{\omega}$ with $\bar{\omega} \triangleq \bar{\delta}+\bar{\gamma}$.

The robot is equipped by a vision system that includes a pinhole camera with limited angles of view $\mathbf{a}$ and $\mathbf{b}$ for $\chi-y$ and $\chi-z$ plane respectively, see Fig.3. The FOV

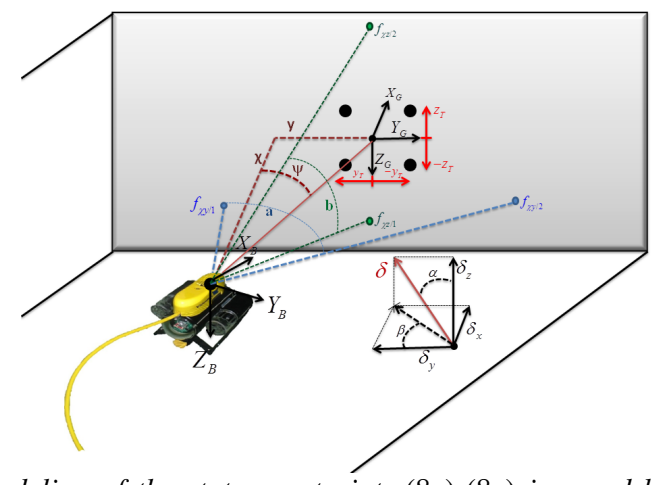

Fig. 3: Modeling of the state constraints (8a)-(8c) imposed by the sensor system and modeling of the external disturbance (5).

constraints of the system can be described as: $\left[-y_{T}, y_{T}\right] \subseteq$ $\left[f_{\chi y / 1}, f_{\chi y / 2}\right]$ and $\left[-z_{T}, z_{T}\right] \subseteq\left[f_{\chi z / 1}, f_{\chi z / 2}\right]$, where $2 y_{T}$ and $2 z_{T}$ are the width and height of the target respectively. Also $\left[f_{\chi y / 1}, f_{\chi y / 2}\right]$ and $\left[f_{\chi z / 1}, f_{\chi z / 2}\right]$ are the camera's fieldof-view on $\chi-y$ and $\chi-z$ plane respectively. Moreover, the vehicle should not exceed the maximum distance $R_{\max }$ at which the target is visible and recognizable to the vision system. These requirements are captured by the state constraint set $X$ of the system, given by:

$$
x_{k} \in X \subset \mathbb{R}^{4}
$$

which is formed by the following constraints:

$$
\begin{gathered}
-y+\chi \tan \left(\psi-\frac{\mathbf{a}}{2}\right)-y_{T} \geq 0, \quad y-\chi \tan \left(\psi+\frac{\mathbf{a}}{2}\right)-y_{T} \geq 0 \\
-z-\chi \tan \left(\frac{\mathbf{b}}{2}\right)-z_{T} \geq 0, \quad z-\chi \tan \left(\frac{\mathbf{b}}{2}\right)-z_{T} \geq 0 \\
R_{\max }^{2}-\chi^{2}-y^{2} \geq 0
\end{gathered}
$$

Furthermore, the control constraint set $V_{\text {set }}$ is assumed to be compact and is given by:

$$
V_{k} \triangleq\left[u_{k}, w_{k}, r_{k}\right] \in V_{\text {set }} \subseteq \mathbb{R}^{3}
$$

The constraints of the input are of the form $|u| \leq \bar{u},|w| \leq \bar{w}$ and $|r| \leq \bar{r}$, therefore we get $\left\|V_{k}\right\| \leq \bar{V}$ where $\bar{V}=\left(\bar{u}^{2}+\right.$ $\left.\bar{w}^{2}+\bar{r}^{2}\right)^{\frac{1}{2}}$ and $\bar{V}, \bar{u}, \bar{w}, \bar{r} \in \mathbb{R}_{\geq 0}$. It can be shown that the nominal system (3) is Lipschitz continuous with Lipschitz constant $0<L_{f}<\infty$. More specifically,

Lemma 1: The nominal model (3), subject to constraints (8a)-(8c) and (9), is locally Lipschitz in $x$ for all $x \in X$, with a Lipschitz constant $L_{f} \triangleq\left(\max \left\{8,8(\bar{u} d t)^{2}\right\}+1\right)^{\frac{1}{2}}$.

\section{B. Control Design and Objective}

The goal is to control the actual system (6) subject to the state constraints of (8a)-(8c) and the control constraints (9), to a desired compact set that includes the desired state $x_{d} \triangleq$ $\left[\chi_{d}, y_{d}, z_{d}, \psi_{d}\right]^{T} \in X$. A predictive controller is employed in order to achieve this task. With the NMPC law the state of the system is proven to converge to the desired set. The design of the ISS predictive controller for system (6) is presented next.
The NMPC consists in solving an Optimal Control Problem (OCP) wrt a control sequence $V_{f}(k)$, for a prediction horizon $N$. The OCP of the NMPC is given as follows:

$$
\begin{aligned}
& \min _{V_{f}(k)} J_{N}\left(x_{k}, V_{f}(k)\right)= \\
& \min _{V_{f}(k)} \sum_{j=0}^{N-1} F(\hat{x}(k+j \mid k), V(k+j \mid k))+E(\hat{x}(k+N \mid K)) \\
& \text { subject to } \\
& \hat{x}(k+j \mid k) \in X_{j}, \quad \forall j=1, \ldots, N-1, \\
& V(k+j \mid k) \in V_{\text {set }}, \quad \forall j=0, \ldots, N-1, \\
& \hat{x}(k+N \mid k) \in \mathscr{E}_{f}
\end{aligned}
$$

Where $\mathscr{E}_{f}$ is the terminal set and $F$ and $E$ are the running and terminal cost function, respectively. The vector $\hat{x}(k+$ $j \mid k)$ denotes the predicted state of the nominal system (3) at sampling time $k+j$ with $j \in \mathbb{Z}_{\geq 0}$. The predicted state is based on the measurement of the state $x_{k}$ of the actual system at sampling time $k$, while applying a sequence of control inputs $\left\{V_{k}, \ldots, V_{k+j-1}\right\}$. Thus:

$$
\hat{x}(k+j \mid k)=f\left(\hat{x}(k+j-1 \mid k), V_{k+j-1}\right)
$$

It holds that $\hat{x}(k \mid k)=x_{k}$. Furthermore, the following preliminary result can be obtained:

Lemma 2: The difference between the actual state $x_{k+j}$ at the time-step $k+j$ and the predicted state $\hat{x}(k+j \mid k)$ at the same time-step, under the same control sequence, is upper bounded by:

$$
\left\|x_{k+j}-\hat{x}(k+j \mid k)\right\| \leq \sum_{i=0}^{j-1}\left(L_{f}\right)^{i} \bar{w}
$$

In Lemma 2, the difference between the actual state trajectory of system (6) and the predicted state trajectory of the nominal system is obtained. To address this divergence, we used a restricted constraint set $X_{j} \subseteq X$ instead of state constraint set $X$ into (10b) (see [13] and [14] for more details). Let the cost function $F(\cdot)$, as well as the terminal cost $E(\cdot)$, to both be of quadratic form, i.e., $F(\hat{x}, V)=$ $\hat{x}^{\top} Q \hat{x}+V^{\top} R V$ and $E(\hat{x})=\hat{x}^{\top} P \hat{x}$, respectively, with $P$, $Q$ and $R$ being positive definite matrices. Particulary we define $Q=\operatorname{diag}\left\{q_{1}, q_{2}, q_{3}, q_{4}\right\}, R=\operatorname{diag}\left\{r_{1}, r_{2}, r_{3}\right\}$ and $P=\operatorname{diag}\left\{p_{1}, p_{2}, p_{3}, p_{4}\right\}$. For the running cost function $F$, it is easy to show that $F(0,0)=0$,

Lemma 3: For the cost function $F(x, V)$ it holds that:

$$
F(x, V) \geq \min \left(q_{1}, q_{2}, q_{3}, q_{4}, r_{1}, r_{2}, r_{3}\right)\|x\|^{2}
$$

The state and input constrained sets are bounded, therefor it can be derived:

Lemma 4: The cost function $F(x, V)$ is Lipschitz continuous in $X \times V_{\text {set }}$, with a Lipschitz constant:

$$
L_{F}=2\left(R_{\max }^{2}+z_{\max }^{2}+\left(\frac{\pi}{2}\right)^{2}\right)^{\frac{1}{2}} \sigma_{\max }(Q)
$$

Where $\sigma_{\max }(Q)$ is the largest singular value of matrix $Q$ and $z_{\max }=R_{\max } \cdot \tan \left(\frac{\mathbf{b}}{2}\right)$ is the maximum feasible value along z-axes.

In order to assert that the NMPC strategy results in a robust stabilizing controller, some stability conditions are stated as follows: 
Assumption 1: For the nominal system (3), there is an admissible positively invariant set $\mathscr{E} \subset X$ such that the terminal region $\mathscr{E}_{f} \subset \mathscr{E}$. Where $\mathscr{E}=\left\{x \in X:\|x\| \leq \varepsilon_{0}\right\}$ and $\varepsilon_{0}$ being a positive parameter.

Assumption 2: We assume that in the terminal set $\mathscr{E}_{f}$, there is a local stabilizing controller $V_{k}=h\left(x_{k}\right) \in V_{\text {set }}$ for all $x \in \mathscr{E}$, and an associated Lyapunov function $E$ such that $E\left(f\left(x_{k}, h\left(x_{k}\right)\right)\right)-E\left(x_{k}\right)+F\left(x_{k}, h\left(x_{k}\right)\right) \leq 0$ for all $x \in \mathscr{E}$.

Assumption 3: The associated Lyapunov function for the terminal region is Lipschitz in $\mathscr{E}$, with Lipschitz constant $L_{E}=2 \varepsilon_{0} \sigma_{\max }(P)$ for all $x \in \mathscr{E}$.

Assumption 4: Inside the set $\mathscr{E}$ we have $E(x)=$ $x^{T} P x \leq \alpha_{\varepsilon}$, where $\alpha_{\varepsilon}=\max \left\{p_{1}, p_{2}, p_{3}, p_{4}\right\} \varepsilon_{0}^{2}>0$. Assuming that $\mathscr{E}=\left\{x \in X_{(N-1)}: h(x) \in V_{\text {set }}\right\}$ and taking a positive parameter $\alpha_{\varepsilon_{f}}$ such that $\alpha_{\varepsilon_{f}} \in\left(0, \alpha_{\varepsilon}\right)$, we assume that the terminal set $\mathscr{E}_{f}=\left\{x \in \mathbb{R}^{3}: E(x) \leq \alpha_{\varepsilon_{f}}\right\}$ is such that $\forall x \in \mathscr{E}, f(x, h(x)) \in \mathscr{E}_{f}$.

\section{Problem Statement}

The solution of the OCP (10a)-(10d) at a time-step $k$ provides an optimal control sequence $V^{*}(\cdot)$. The classic framework of the MPC consists in applying to the system only the first control vector, i.e., $V^{*}(k \mid k)$ and to discard all the remaining elements of the sequence. At the next time-step $k+1$, new state measurements are received and the whole procedure is repeated again. This is iteratively repeated until the system has reached to the desired terminal set. However, the self-triggering framework suggests that a portion of the computed control sequence may be applied to the system and not only the first vector. Suppose a triggering instant $k_{i}$. The control sequence that is applied to the plant is of the form

$$
\left\{V^{*}\left(k_{i} \mid k_{i}\right), V^{*}\left(k_{i}+1 \mid k_{i}\right), \ldots V^{*}\left(k_{i}+d_{i} \mid k_{i}\right)\right\}
$$

for all $d_{i} \in\left[0, k_{i+1}-k_{i}\right]$, where $k_{i+1}$ is the next triggering instant. During the time interval $\left[k_{i}, k_{i+1}\right)$ the control law is applied to the plant in an open-loop fashion i.e., no state measurements from the system are received. A question that naturally arises is how large this time interval can be? Notice, though, the smallest time interval is obviously 1 , that is if $k_{i+1}=k_{i}+1$ and that the largest time interval is $N-$ 1. The self-triggered strategy that will be presented later in this paper, answers to this question and provides sufficient conditions for finding the recalculation periods, or in other words sufficient conditions for triggering the computation of the NMPC law. This leads us to the statement of the problem treated in this paper:

Problem Statement 1: Consider the system (6) that is subject to constraints (7) and (9). The objective is $(i)$ to design a feedback control law provided by (10a)-(10d) such that the system (6) converges to the terminal constraint set and $(i i)$ to find a mechanism to decide when the next control update should be.

\section{Stability AnAlysis OF SElF-TRIGGERING NMPC FRAMEWORK}

It can be proven that the closed-loop scheme (6)-(14) is ISS wrt the disturbances, [15]. Moreover, through the ISS analysis it is possible to reach to a self-triggering mechanism which provides the triggering instants. The proof of the stability of a system under the NMPC consists in guaranteeing (i) the feasibility property and (ii) the convergence property of the closed-loop system.

We begin by showing that initial feasibility implies feasibility afterwards. Consider two successive triggering events $k_{i}$ and $k_{i+1}$. A feasible control sequence $\tilde{V}(k+j \mid k+m)$, for time steps $m=0, \ldots, N-1$, based on the optimal solution at the time-step $k-1$, is given by:

$$
\begin{aligned}
& \tilde{V}(k+j \mid k+m)= \\
& \left\{\begin{aligned}
V^{*}(k+j \mid k-1) & \text { for } j=m, \ldots, N-2 \\
h(\hat{x}(k+j \mid k+m)) & \text { for } j=N-1, \ldots N+m-1
\end{aligned}\right.
\end{aligned}
$$

From the feasibility of the initial sequence at time-step $k-1$ and with the help of Assumption 2, it follows that for $m=$ $0, \ldots N-1$ we have $\tilde{V}(k+j \mid k+m) \in V_{\text {set }}$. It could be proven that $\hat{x}(k+N \mid k+m) \in \mathscr{E}_{f}$ for all $m=0, \ldots, N-1$.

The state convergence of the perturbed system to a desired terminal set is going to be shown in the sequel. A proper value function must be shown to be decreasing in order to prove stability of the closed-loop system. The optimal cost will be used as a natural Lyapunov function candidate for the stability analysis.

At the time-step $k-1$, the optimal cost is denoted as $J^{*}(k-1)$, which is evaluated under the optimal control sequence. Analogously, the optimal cost at a time-step $k+m$ with $m \in[0, N-1]$ is denoted as $J^{*}(k+m)$. Now let $\tilde{J}_{N}(k+m)$ to denote the "feasible" cost, evaluated from the control sequence (15). This "feasible" cost will help us to obtain the difference $J^{*}(k+m)-J^{*}(k-1)$. In particular the difference between the feasible sequence at time-step $k+j$ and the optimal cost at time $k-1$ using (15) is given by:

$$
\begin{aligned}
& \Delta J_{m}=\tilde{J}_{N}(k+m)-J_{N}^{*}(k-1)= \\
& \leq\left(L_{E} \cdot\left(L_{f}\right)^{(N-(m+1))}+L_{F} \cdot \sum_{i=0}^{N-(m+2)}\left(L_{f}\right)^{i}\right) \bar{w} \\
& -\sum_{i=-1}^{m-1} \min \left(q_{1}, q_{2}, q_{3}, q_{4}, r_{1}, r_{2}, r_{3}\right)\|\hat{x}(k+i \mid k-1)\|
\end{aligned}
$$

The optimality of the solution yields

$$
J_{N}^{*}(k+m)-J_{N}^{*}(k-1) \leq \tilde{J}_{N}(k+m)-J_{N}^{*}(k-1)
$$

The Lyapunov function $J^{*}(\cdot)$ has been proven to be decreasing, thus the closed-loop system converges to a compact set $\mathscr{E}_{f}$, where it is ultimately bounded, due to Assumption 4.

\section{A. The Self-triggered Framework}

In this section the self-triggering mechanism is going to be presented. Consider that at time-step $k_{i}$ an event is triggered. The next control update time $k_{i+1}$ is considered to be unknown and should be found. In particular, the next control update time $k_{i+1} \triangleq k_{i}+d_{i}$ should be such that the closed-loop system does not loose any of its desired 
properties. Thus, we still need the Lyapunov function $J^{*}(\cdot)$ to be decreasing. Given (16) and (17), for a triggering instant $k_{i}$ and a time-step $d_{i}$ with $d_{i} \in[1, N-1]$ we get:

$$
\begin{aligned}
& J^{*}\left(k_{i+1}\right)-J^{*}\left(k_{i}\right) \\
& \leq\left(L_{E} \cdot\left(L_{f}\right)^{\left(N-d_{i}\right)}+L_{F} \cdot \sum_{i=0}^{N-\left(d_{i}+1\right)}\left(L_{f}\right)^{i}\right) \bar{w}-L_{Q}\left(d_{i}\right)
\end{aligned}
$$

where:

$L_{Q}\left(d_{i}\right)=\sum_{i=1}^{i=d_{i}} \min \left(q_{1}, q_{2}, q_{3}, q_{4}, r_{1}, r_{2}, r_{3}\right)\|\hat{x}(k+i \mid k-1)\|$

The time instant $d_{i}$ should be such that:

$$
\left(L_{E} \cdot\left(L_{f}\right)^{\left(N-d_{i}\right)}+L_{F} \cdot \sum_{i=0}^{i=N-\left(d_{i}+1\right)}\left(L_{f}\right)^{i}\right) \bar{w} \leq \sigma \cdot L_{Q}\left(d_{i}\right)
$$

with $0<\sigma<1$ and Plugging (19) to (18), we get:

$$
J^{*}\left(k_{i+1}\right)-J^{*}\left(k_{i}\right) \leq(\sigma-1) L_{Q}\left(d_{i}\right)
$$

This suggests that provided $0<\sigma<1$, the convergence property is still guaranteed. Thus, the next control update time should be triggered when (19) is violated. The condition (19) should be checked for each consecutive time-step, i.e., for $d_{i}=1,2, \ldots$. The time-step that this condition does no longer holds should be the next triggering instant $k_{i+1}$. Notice that the time-step $k_{i+1}$ can be found beforehand at time $k_{i}$, i.e, this is a self-triggering mechanism. Moreover, it should be pointed out that the term $L_{Q}\left(d_{i}\right)$ includes only predictions of the nominal system that is forming a predicted sequence and that can also be computed by forward integration of (3) for time-steps $d_{i} \in[1, N-1]$.

Next we describe the self-triggering mechanism. At time $k_{i}$ a control update is triggered and a control trajectory for $\left[k_{i}, k_{i}+N-1\right]$ is provided. The solution of (19) will provide the next update time $k_{i+1}$. During the time interval $k \in$ $\left[k_{i}, k_{i+1}\right)$ the control trajectory $V^{*}\left(k_{i}+d_{i} \mid k_{i}\right)$ is applied to the plant in an open-loop fashion. Next, at time-step $k_{i+1}$ the optimal control problem of the NMPC is solved again using the current state measurement $x\left(k_{i+1}\right)$ as the initial state. The controller follows this procedure until the system converges to the terminal constraint set.

\section{EXPERIMENTS}

The aim of this section is to show the efficacy of the proposed self-triggered NMPC controller to perform a real experiment. An attitude stabilization of an underwater vehicle was performed in real-time. Notice that the water is a significant source of disturbance in this experiment.

\section{A. System Components}

The ROV used is a 3-DOF (VideoRay PRO, VideoRay LLC, Fig.2), equipped with three thrusters, a control unit, and a CCD camera. The control unit is connected with a Personal Computer (PC). The image dimensions are $640 \times 480$ pixels. A target is located on an aluminium plate which is fixed inside the tank. The system software is implemented using

the Robotics Operating System (ROS, http://www.ros.org). The target detection and vehicle localization are shown in Fig.4.

a) Initial configuration
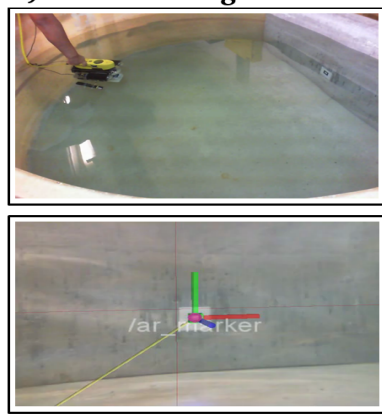

Fig. 4: Experimental setup. (a) The initial configuration. Vehicle's view (b) The desired pose of the vehicle. Vehicle's view.

\section{B. Experimental Results}

The goal of the experiment is the attitude stabilization of the vehicle at the desired position. The first experiment using the classical (time triggering) NMPC, will be compared to the second experiment where the Self-Triggering NMPC proposed in this paper is used. The initial and the desired position of the robot wrt the target frame is $\left[\chi_{i n}, y_{i n}, z_{i n}, \psi_{i n}\right]=[-1.2,0.45,0.1,0.401]$ and $x_{d}=$ $\left[\chi_{d}, y_{d}, z_{d}, \psi_{d}\right]^{\top}=[-0.6,0.0,0.0,0.0]$ respectively. The sampling time of this experiment is equal to $d t=0.15 \mathrm{sec}$. The maximum translational and angular velocity of the vehicle in surge, heave and yaw direction are $\bar{u}=0.2 \mathrm{~m} / \mathrm{sec}, \bar{w}=$ $0.3 \mathrm{~m} / \mathrm{sec}$ and $\bar{r}=0.3 \mathrm{rad} / \mathrm{sec}$, respectively. The matrices $Q$, $R$ and $P$ are defined as $Q=\operatorname{diag}(0.5,4.5,4.5,0.1), R=$ $\operatorname{diag}(0.17,0.1,1)$ and $P=\operatorname{diag}(1,1,1,1)$, respectively.
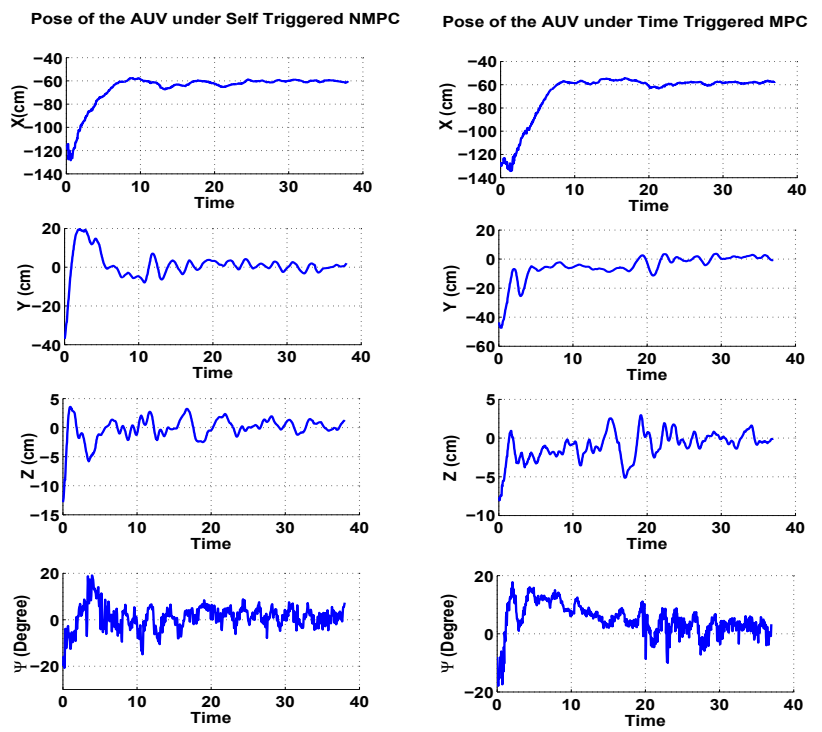

Fig. 5: The state of the robot wrt the target frame. (a) Self TriggeredMPC. (b) Time Triggered MPC

In Fig. 5 the states evolution of the system about $x, y$ and $\psi$ in both experiments are depicted. The states of the system are converging to a set around the desired position in both 
cases. It can be witnessed that the performances in the case of self triggering are better (or the same) comparing to the classical case. In both cases, all the constraints are satisfied and the robot have reached the desired position wrt the target.

In Fig. 6 the triggering instants in the case of self triggering are captured. When the vertical axis has the value 1 , the NMPC is triggered, the image is processed and the state vector is calculated, consequently the optimization algorithm is running and a new control input sequence is computed. For value 0, the control law is implemented on the system in an open-loop fashion using the rest of the last computed control input sequence, thus no optimization and no image processing is running.

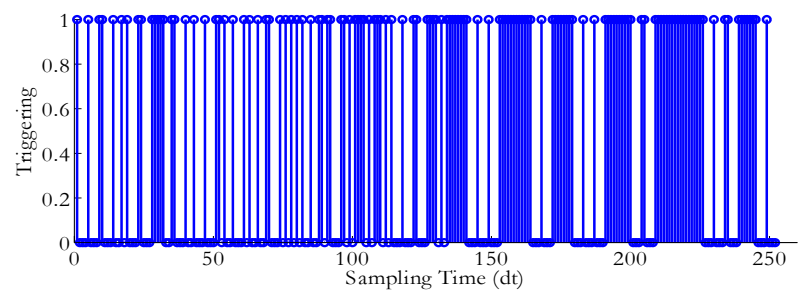

Fig. 6: The triggering instants in Self triggered NMPC . For value 1, the NMPC is triggered. For value 0 the control law is implemented on the robot in an open-loop fashion.

The computational time in a triggering instance is about $0.1 \mathrm{sec}$ while in the case of the open loop control is being reduced to $0.0002 \mathrm{sec}$. This is happening because neither the image processing nor the optimization algorithm are running between two triggering instances in the case of self triggered control. This is very important in the case of underwater vehicle where the energy sources (batteries) are very limited and the recharging is very difficult and time consuming. Finally in Fig.7 the control inputs are depicted. It can be seen that no violation of the constraints took place.
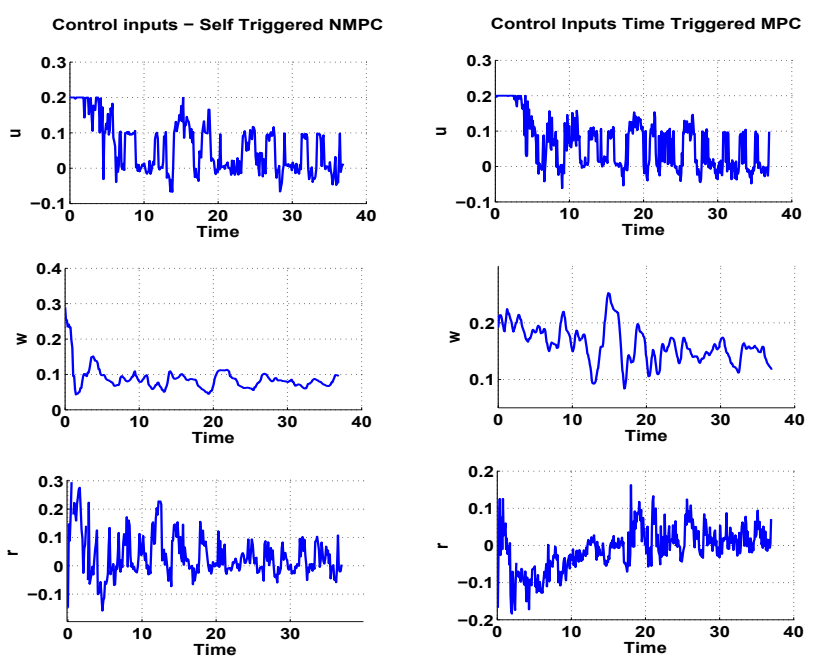

Fig. 7: Control inputs. (a) Self Triggered MPC. (b) Time Triggered $M P C$.

\section{Summary AND Future Work}

In this paper, a Self-triggered Visual Servo Model Predictive Control Scheme for Underwater Robotic Vehicles is presented. The main idea is to trigger and run the NMPC only when it is needed and not periodically as in the case of the classic NMPC schemes. During the inter-sampling instants the control sequence from the NMPC is applied to the system in an open-loop fashion, i.e., neither the image processing nor the optimization algorithm are running between two triggering instances. In this paper sufficient conditions for triggering were provided along with an experiment that shows the effectiveness of the proposed framework comparing to the classical time triggered NMPC. Future work will involve extention of the proposed visual servo controller taking into consideration the obstacles in the trajectory of the robot.

\section{ACKNOWLEDGMENT}

This work was supported by the EU funded project PANDORA: Persistent Autonomy through learNing, aDaptation, Observation and ReplAnning, FP7288273, 20122014.

\section{REFERENCES}

[1] F. Chaumette and S. Hutchinson, "Visual servo control. i. basic approaches," IEEE Robotics and Automation Magazine, vol. 13, no. 4, pp. 82-90, 2006.

[2] F. Chaumette and S. Hutchinson, "Visual servo control, part ii: Advanced approaches," IEEE Robotics and Automation Magazine, vol. 14, no. 1, pp. 109-118, 2007.

[3] S. Heshmati-alamdari, G. K. Karavas, A. Eqtami, K. Drossakis, and K. J. Kyriakopoulos, "Robustness analysis of model predictive control for constrained image-based visual servoing," Proceedings of the 2014 IEEE International Conference on Robotics and Automation, 2014.

[4] P. Tabuada, "Event-triggered real-time scheduling of stabilizing control tasks," IEEE Transactions on Automatic Control, vol. 52, no. 9, pp. 1680-1685, 2007.

[5] A. Anta and P. Tabuada, "To sample or not to sample: self-triggered control for nonlinear systems," IEEE Transactions on Automatic Control, vol. 55, no. 9, pp. 2030-2042, 2010.

[6] D. Lehmann and J. Lunze, "Event-based control: A state feedback approach," European Control Conference, pp. 1716-1721, 2009.

[7] X. Wang and M. Lemmon, "Self-triggered feedback control systems with finite-gain L2 stability," IEEE Transactions on Automatic Control, vol. 45, no. 3, pp. 452-467, 2009.

[8] A. Eqtami, S. Heshmati-alamdari, D. V. Dimarogonas, and K. J. Kyriakopoulos, "Self-triggered model predictive control for nonholonomic systems," European Control Conference, 2013. to appear.

[9] S. Durand, J. Guerrero-Castellanos, N. Marchand, and W. GuerreroSnchez, "Event-based control of the inverted pendulum: Swing up and stabilization," Control Engineering and Applied Informatics, vol. 15, no. 3, pp. 96-104, 2013.

[10] J. T. Guzman, J. Guerrero-Castellanos, S. Durand, N. Marchand, and R. L. Maya, "Event-based lqr control for attitude stabilization of a quadrotor," 15th IFAC Latinamerican Control Conference, 2012.

[11] T. Fossen, "Guidance and control of ocean vehicles," Wiley, New York, 1994.

[12] D.Panagou and K. J. Kyriakopoulos, "Control of underactuated systems with viability constraints," Proc. of the 50th IEEE Conference on Decision and Control and European Control Conference, pp. 54975502, 2011.

[13] D. L. Marruedo, T. Alamo, and E. Camacho, "Input-to-state stable mpc for constrained discrete-time nonlinear systems with bounded additive uncertainties," 41st IEEE Conf. Decision and Control, pp. 4619 - 4624, 2002.

[14] G.Pin, L. Magni, T. Parisini, and D. Raimondo, "Robust recedinghorizon control of nonlinear systems with state dependent uncertainties: an input-to-state approach," American Control Conference, pp. 1667 - 1672, 2008.

[15] Z.-P. Jiang and Y. Wang, "Input-to-state stability for discrete-time nonlinear systems," Automatica, vol. 37, no. 6, pp. 857-869, 2001. 